NOTULES HYDRAULIQUES

\title{
Pour servir à l'édification d'une hydraulique géométrique
}

\section{Basis for setting up geometrical hydraulics}

Duns le cas simple d'une cheminée d'équilibre placée en tète d'une conduite d̀ caractéristique unique l'autear propose une transposition géométrique de l'évolution du coup de bélier d'onde ou de l'oscillation en masse.
For the simple case of a surge-lanli at the head of a penstock with a single characteristic, the athor proposes a geometrical transposition of the evolution of the wave waterhammer or of mass oscillation.
L'analyste qui vient de metlre le point final à un long calcul pour démontrer une proposition quelconque est toujours très heureux lorsqu'il peut trouver une démonstration " par la géométrie pure $»$ tellement les raisonnements nets et "sans bavure » de cette science surpassent les ealculs longs et pénibles de i'analyse.

II ne semble pas qu'il y ait en hydraulique beaucoup de place pour les démonstrations géométriques. Cependant, en cherchant bien, on pourait peut-être en trouver.

Prenons par exemple le probleme de la chemince d'équilibre placé à l'origine d'une conduile à caractéristique unique.

Si $\tau=2 \mathrm{~L} / a$ est la période d'aller et retour, on connail la construction classique qui, dans le diagramme hauteur-vitesse, permet de construire l'état à l'instant $t+\tau$ à partir de l'état comnu à linstant $t$.

Soient :

At le point caractéristique à l'instant $t$, ef :

$B_{t+\tau}$ le point caractéristique à l'instant $t+\tau$
$B_{t+\tau}$ est sur la droite caractéristique de pente $a / g$ réfléchie sur l'horizontale du reservoir d'extrémité.

Be même $B_{t+\tau}$ se trouve sur la caractéristique de la cheminée qui est une droite de pente - ( L w) / (a s) (w section de la conduite, s section de lat chemince passant par le symétrique $A^{\prime}$ par rapport à l'axe des ritesses du point $A$. Il revient au même de dire que $B$ est sur une droite réléchie sur l'axe des vitesses.

Ces droites gardent tout au long de l'épure des pentes constantes.

On peul fort bien fixer l'échelle des vitesses par rapport à celle des hauteurs de telle sorte que ces droites soient perpendiculaires, l'angle de réflexion étant alors égal pour les deux caractéristiques.

Examinons dans ce cas la construction :

Menons les normales aux axes MP et NP, ces normales se rencontrant en $P$. 
Les angles $\widehat{\mathrm{MBN}}$ et $\widehat{\mathrm{MAN}}$ étant droits, les six points $O N B P A M$ sont sur un mème cercle.

Joignons B O, PO et A O.

L'angle $\widehat{A O B}$ est égal à $\widehat{A M B}$ et $\mathrm{PO}$ est la bissectrice de $\widehat{\mathrm{AOB}}$.

Par suite :

$$
\mathrm{AO}=\mathrm{OB}
$$

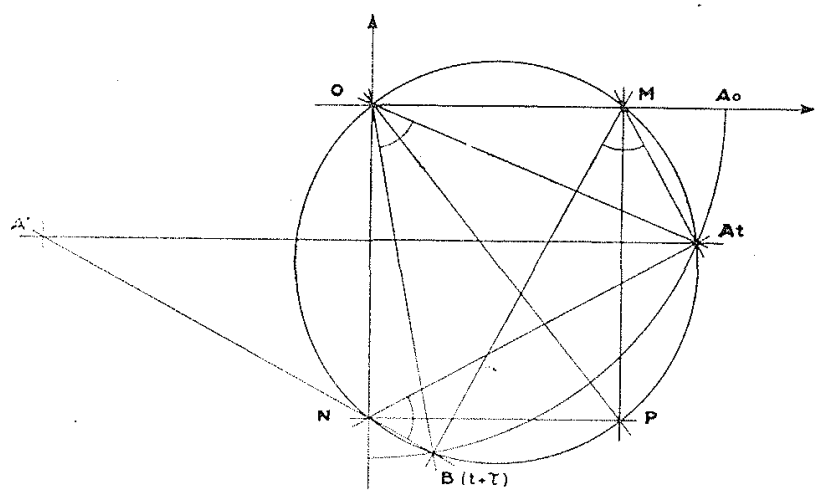

Le point $B_{\text {th- }}$ se trouve done sur un cerele de centre $\mathrm{O}$ et de rayon $\mathrm{OA}$.

On en déduit done que le lieu du point $B$ pendant une période allant de $K \tau$ à $(K+1) \tau(K$ entier quelconque), se déduit du lieu de A pendant la période précédente allant de $(K-1) \tau$ à $K$ 。 par une rotation définie par l'angle A M B.

\section{Notamment :}

a l'instant 0 , A est en $A_{0}$ tel que $O A_{0}=v_{0}$ a l'instant 1 , $A$ est en $A_{1}$ tel que $O A_{1}=D_{0}$
De l'instant 0 à l'instant 1 , on sait que A reste sur la droite $A_{0} A_{1}$.

Par conséquent, l'épure caractéristique de l'étal en $A$ est un polygone régulier de centre $O$ inscrit dans un cercle de rayon $v_{0}$; chaque côté ayant pour angle au centre $\widehat{A M B}$; il en résulte que ce polygone ne sera fermé que si $\widehat{A} M$ B $B$ est un sous-multiple de $2 \mathrm{~K} \pi$ ( $\mathrm{K}$ entier quelconque).

La démonstration précédente n'est valable que dans la mesure ou nous avons pu écrire pour l'équation de continuité :

$$
-\mathrm{S}\left(h_{2}-h_{1}\right)=\omega\left(\frac{v_{1}+v_{2}}{2}\right)==\frac{\mathrm{L} \omega}{a}\left(v_{1}+v_{2}\right)
$$

e'ost-a-dire (que pour une valeur de la période = suffisamment faible. D'aulre part, il a été fait évidemment abstraction de toute perte de charge.

Si cette période diminue encore, nous voyons que le polygone tend vers le cercle de rayon $v_{\text {, }}$ et on retombe sur le résultat bien connu de l'oscillation en masse :

$$
\begin{aligned}
& h=h_{0} \sqrt{\frac{\mathrm{L}}{g s}} \sin \sqrt{\frac{g s}{\mathrm{~L}}} t \\
& D=v_{0} \cos \sqrt{\frac{g s}{\mathrm{~L}_{1}(1)}} t
\end{aligned}
$$

équations dont la représentation graphique pour une échelle convenable est bien un cercle.

Ceci confirme, dans le cas présent, ce que nous savons dejà, à savoir que l'oscillation cn masse n'est qu'un cas particulier du coup de bélier d'onde correspondant à une célérité a infinie.

\section{DUBin,}

Ingenieur des Arts et Manufactures.

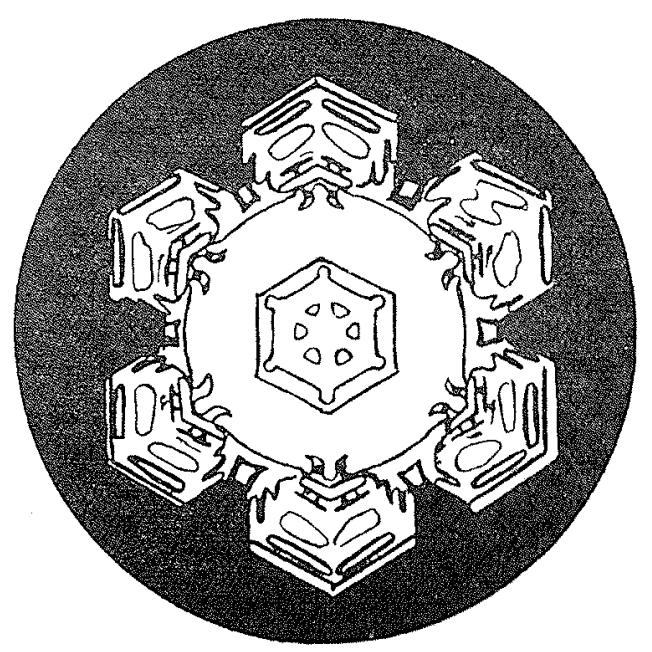

